\title{
Method of axial adjustment for precessional transmissions
}

\author{
Ion Bostan, Sergiu Mazuru and Maxim Casian* \\ Technical University of Moldova, 9, Studenţilor str., Chisinau, Republic of Moldova
}

\begin{abstract}
Axial adjustment method for precessional transmissions includes compensation error calculation, determination compensator groups, achieved in the form of base and auxiliary rings. The compensator has on the base ring axial canals in which can be placed clamping bolts but the auxiliary ring has axial holes for the screws. The auxiliary ring is formed from increasing sectors - the distance between auxiliary ring stairs and front side of body, from decreasing sectors - the distance between base ring and bottom place, and the closure element - the clearance between gear body and the front surface of the base ring. According to the method of group interchangeability is determined the deviation value of the upper and lower group tolerance and the clearance between lid and body. Then rotating one of the rings is ensured the dimension of the compensator which must match to compensation clearance value and then the compensator is adjusted to required value.
\end{abstract}

\section{Introduction}

This method of adjustment of axial movements without thread can be used to adjust clearance in gear, especially in gearboxes. From [1] is known the adjustment process of axial clearance, in which from chains of dimensions are determined the amount of compensation, the number of fixed compensators steps and their size. Then in assembling process is measured the axial clearance between front surface of the lid and the bearing, is chosen compensation gaskets size and is installed on the closure element of the chain. The disadvantage of this method of clearance compensation is high workmanship of assembling; because of the collecting complexity for the required compensation rings and also the execution complexity of the rings (less than $0.1 \mathrm{~mm}$ ). This method has a disadvantage namely cannot be used to adjust conic gear. The purpose of this method is to decrease the assembling costs of the frontal surfaces of machines nodes, which contain precessional gearing, and to increase of adjustment precision for axial clearance.

\section{Compensator for axial adjustment}

Intended purpose can be achieved in that axial adjustment method for precessional transmissions include calculation of compensation error, determination compensator groups

\footnotetext{
*Corresponding author: max_casian@yahoo.com
} 
achieved in the form of ring formed as a minimum of four places and auxiliary ring (Figure 11), which has on the front side stairs places diametrically opposed. The compensator is characterized that the base ring has axial canals in which can be placed clamping bolts but the auxiliary ring has axial holes for the passage of screws. The auxiliary ring is formed from increasing sectors - the distance between auxiliary ring stairs and front side of body, from decreasing sectors - the distance between base ring and the bottom place, and the closure element - the clearance between gear body and the front surface of the base ring. According to the method of group interchangeability is determined the deviation value of the upper and lower group tolerance and the clearance between gear lid and gear body. Then rotating one of the rings is ensured the dimension of the compensator which must match to the compensation clearance value and then the compensator is adjusted to the required value.

Also the process of axial adjustment can be achieved using another compensator. The process of axial adjustment consists from auxiliary ring of compensator which has axial channels and the base ring with axial holes.

The technical positive result ensured by all the characteristics of the process consist of decreasing the assembling costs of the frontal surfaces of machines nodes, which contain precessional gearing, and of increasing of adjustment precision for axial clearance.

The reducer consist: shell 1, lid 2, compensator 3, driving shaft 4, fixed wheel, satellite 6 with crowns 7 and 8, driven shaft 10, bearing 11, bushing 12, bearing 13 and lid 14 [3]. As a rule the gearbox before final assembly has two subassemblies: the one on the right (Figure1) and the one on the left (Figure 2). In the left subassembly we have body parts 1, mobile wheel 9, driven shaft 10, bearings 11 and 13, bushing 12 and the lid 14 . The right subassembly has lid 2, compensator 3, driving shaft, the fixed wheel, satellite 6 and crowns 7 and 8 . Assembly is made by insertion of the left side in the right side. When assembling these two parts by moving in the axial direction the left side is ensured centering of the right bearing of driving shaft in the cylindrical seat of the driven shaft. The displacement in axial direction ends when mobile wheel 9 came in contact with crown 8 of the satellite 6 . It is also necessary to center the body 1 with the lid 2 during the axial displacement of the left side to the right. Finally it is necessary to adjust the clearance between crown 7 and gear wheel 2.

\section{Dimensional chains calculation}

Realization of the process we will examine by the example of precessional gear (Figure 4).

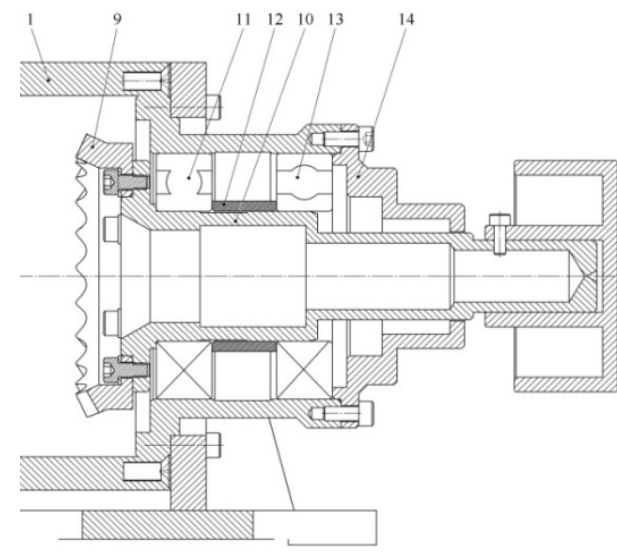

Fig. 1. Right subassembly.

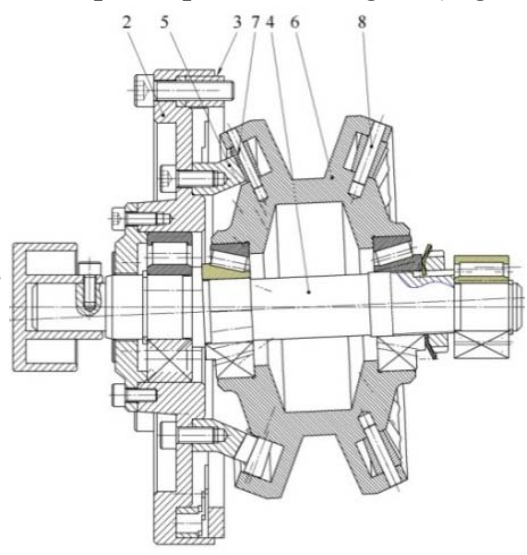

Fig. 2. Left subassembly. 
From dimensional chain (Figure 5) by the method of total interchangeability [2] is determined the value of the closure element $A_{\Delta}=\sum_{i=1}^{n} T A_{i}$;

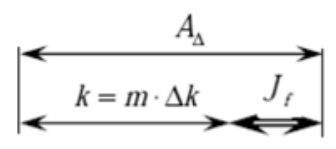

Fig. 3. Dimensional chain for compensator to ensure functional clearance.

The proper functioning of the gear unit is done under a summary functional clearance in the both gear couplings - $J_{f}$. From dimensional chain (Figure 3 ) it is seen that will be compensated the maximum possible value:

$$
A_{\max }^{c o m p}=A_{\Delta}-J_{f}
$$

Compensation in cases of concrete construction of the reducer is made at the value by selecting the compensator ring steps 3 (Figure 4).

The value $A_{\max }^{\text {comp }}$ is divided on the compensator steps with height $\Delta k$ in number $m$.

To avoid overcompensation and appearing of tightening in gear couplings, the value $\Delta k$ should not surpass the functional value of clearance $J_{f}, \Delta k=<J_{f}$ [4]. The value $\Delta k$ depends on the requirements stringency to the values range of the functional clearance $J_{f}$ and determines the functional clearance variation due to discretized character of compensation in limitations of one step of compensation.

The calculated number of steps:

$$
m_{c}=A_{\max }^{c o m p} / \Delta k-1
$$

(minus 1, because compensation is made using the ,body” of compensation ring, i.e. step „zero"). The integer number of steps $m$ is obtained by rounding to the higher integer number (to ensure ${ }^{\Delta k=<J_{f}}$ ). The value of one step:

$$
\Delta k_{c}=A_{\max }^{c o m p} / m+1
$$

The number of step on which is made the compensation $T R$ represents the integer part of calculated number of step:

$$
T R_{c}=A^{c o m p}-j_{f} / \Delta k_{c} .
$$




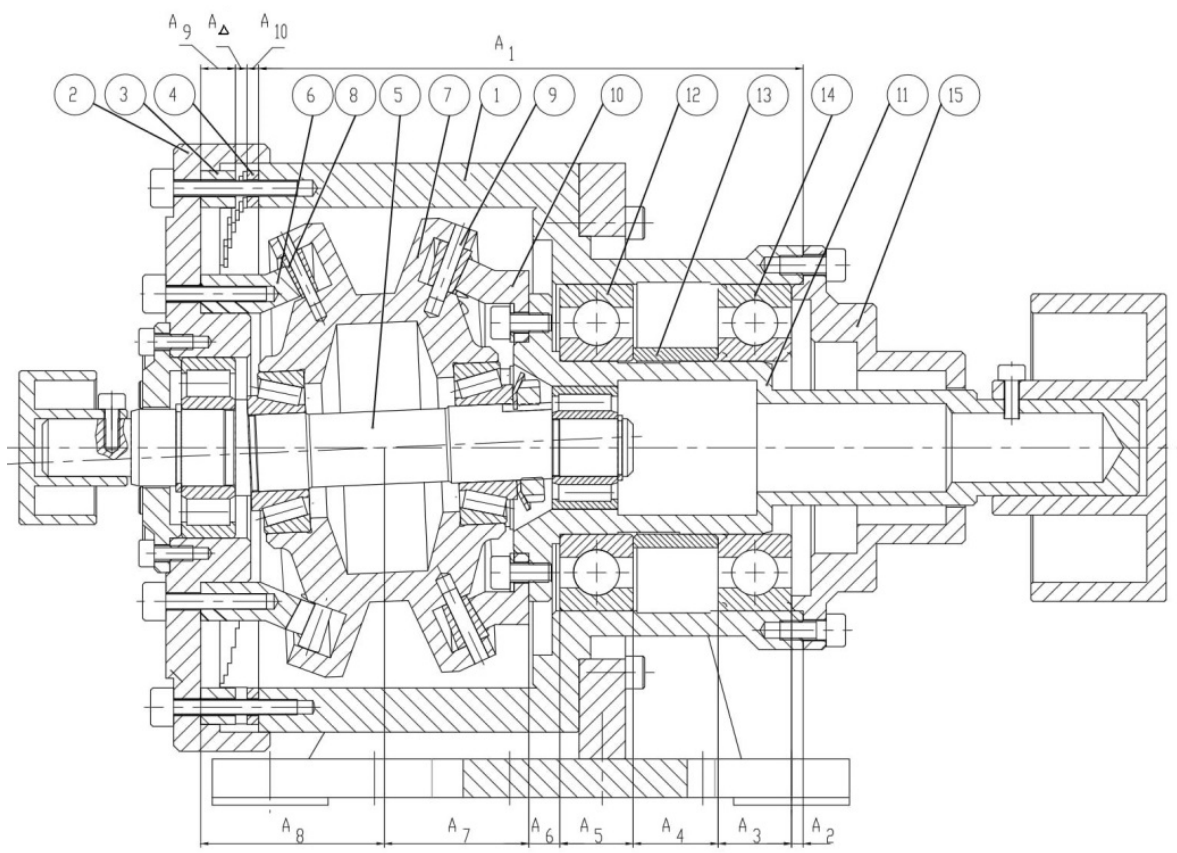

Fig. 4. Axial section for reducer with one compensator.

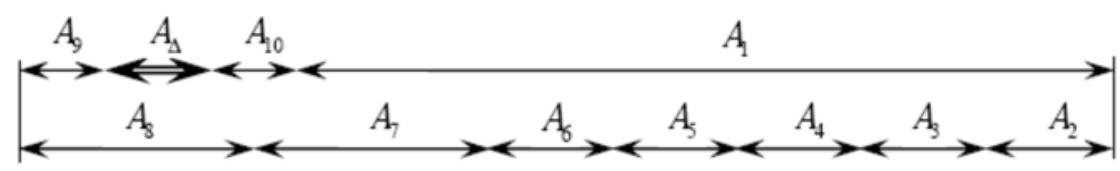

Fig. 5. Dimensional chain for reducer.

Functional clearance after compensation:

$$
J_{f c}=A^{c o m p}-T R \cdot \Delta k_{c}
$$

Deviation of functional clearance:

$$
\Delta J_{f}=J_{f c}-J_{f}
$$

Maximum possible clearance:

$$
J_{f \max }=J_{f}+\Delta k
$$

Example. Summary value of clearance in both functional gear couplings $J_{f}=0.1 \mathrm{~mm}$ :

$$
\begin{gathered}
A_{\Delta}=\sum_{i=1}^{n} T A_{i}=0.78 \mathrm{~mm} ; \\
A_{\max }^{c o m p}=0.78-0.1=0.68 \mathrm{~mm}
\end{gathered}
$$


We choose the option $\Delta k=J_{f}=0.1 \mathrm{~mm}$. The compensator steps number $m=6$ that is the integer part of:

$$
m_{c}=\frac{A_{\max }^{\text {comp }}}{\Delta k}-1=\frac{0.68}{0.1}-1=5.8
$$

The results of the calculations are given in the Table 1 , and for the case $\Delta k=0.07 \mathrm{~mm}$. The schema of compensator steps executed to IT9 is shown in Figure 6. In precessional reducers, especially with two gear couplings, the elements number in dimensional chain is high, so the total clearance $A_{\Delta}=\sum_{i=1}^{n} T A_{i}$ and the maximum compensation $A_{\max }^{\text {comp }}$ get to the values of a few millimeters. Therefore, the number of compensation steps becomes very high.

Table 1. Obtained values.

\begin{tabular}{|c|c|c|c|c|}
\hline \multicolumn{2}{|c|}{$J_{\rho s}=0.1 \mathrm{~mm} \Delta k=0.1 \mathrm{~mm}$} \\
\hline \multirow{2}{*}{$\begin{array}{c}\text { Compensated } \\
\text { dearance }\end{array}$} & $\begin{array}{c}\text { Step of } \\
\text { compenstion }\end{array}$ & \multicolumn{2}{c|}{$\begin{array}{c}\text { Functional } \\
\text { dearnce }\end{array}$} \\
\cline { 2 - 5 } & Calc. & Adopt & Value & Dev. \\
\hline$A^{\text {somp }}$ & $T R_{c}$ & $T R$ & $J_{\rho}$ & $\Delta J_{f}$ \\
\hline 0.1 & 0 & 0 & 0.1 & 0 \\
\hline 0.11 & 0.1 & 0 & 0.11 & 0.01 \\
\hline 0.19 & 0.9 & 0 & 0.19 & 0.09 \\
\hline 0.2 & 1 & 1 & 0.1 & 0 \\
\hline 0.21 & 1.1 & 1 & 0.11 & 0.01 \\
\hline 0.29 & 1.9 & 1 & 0.19 & 0.09 \\
\hline 0.3 & 2 & 2 & 0.1 & 0 \\
\hline 0.31 & 2.1 & 2 & 0.11 & 0.01 \\
\hline 0.39 & 2.9 & 2 & 0.19 & 0.09 \\
\hline 0.4 & 3 & 3 & 0.1 & 0 \\
\hline 0.41 & 3.1 & 3 & 0.11 & 0.01 \\
\hline 0.49 & 3.9 & 3 & 0.19 & 0.09 \\
\hline 0.5 & 4 & 4 & 0.1 & 0 \\
\hline 0.51 & 4.1 & 4 & 0.11 & 0.01 \\
\hline 0.59 & 4.9 & 4 & 0.19 & 0.09 \\
\hline 0.6 & 5 & 5 & 0.1 & 0 \\
\hline 0.61 & 5.1 & 5 & 0.11 & 0.01 \\
\hline 0.68 & 5.8 & 5 & 0.18 & 0.08 \\
\hline
\end{tabular}

\begin{tabular}{|c|c|c|c|c|}
\hline \multicolumn{5}{|c|}{$J_{f k}=0.1 \mathrm{~mm} \quad \Delta k=0.07 \mathrm{~mm}$} \\
\hline \multirow{2}{*}{$\begin{array}{l}\text { Compensated } \\
\text { dearance }\end{array}$} & \multicolumn{2}{|c|}{$\begin{array}{c}\text { Step of } \\
\text { compens ation }\end{array}$} & \multicolumn{2}{|c|}{$\begin{array}{c}\text { Functional } \\
\text { clearance }\end{array}$} \\
\hline & Calc. & Adopt. & $\mathrm{V}_{2} \mathrm{l}$. & Dev: \\
\hline$A^{\text {somp }}$ & $T R_{c}$ & $T R$ & $J_{s}$ & $\Delta_{f}$ \\
\hline 0.1 & 0.00 & 0 & 0.1 & 0 \\
\hline 0.11 & 0.14 & 0 & 0.11 & 0.01 \\
\hline 0.19 & 1.29 & 1 & 0.12 & 0.02 \\
\hline 0.2 & 1.43 & 1 & 0.13 & 0.03 \\
\hline 0.21 & 1.57 & 1 & 0.14 & 0.04 \\
\hline 0.29 & 2.71 & 2 & 0.15 & 0.05 \\
\hline 0.3 & 2.86 & 2 & 0.16 & 0.06 \\
\hline 0.31 & 3.00 & 3 & 0.1 & 0 \\
\hline 0.39 & 4.14 & 4 & 0.11 & 0.01 \\
\hline 0.4 & 4.29 & 4 & 0.12 & 0.02 \\
\hline 0.41 & 4.43 & 4 & 0.13 & 0.03 \\
\hline 0.49 & 5.57 & 5 & 0.14 & 0.04 \\
\hline 0.5 & 5.71 & 5 & 0.15 & 0.05 \\
\hline 0.51 & 5.86 & 5 & 0.16 & 0.06 \\
\hline 0.59 & 7.00 & 7 & 0.1 & 0 \\
\hline 0.6 & 7.14 & 7 & 0.11 & 0.01 \\
\hline $0.6 !$ & 7.29 & 7 & 0.12 & 0.02 \\
\hline 0.68 & 8.29 & 8 & 0.12 & 0.02 \\
\hline
\end{tabular}

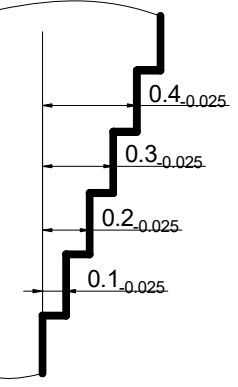

Fig. 6. Schema with compensator stairs (example). 
The number of steps becomes big and in case when the requirements for the precision of functional clearance are high, i.e. $\Delta k<J_{f}$.

Is appropriate to use two compensators, one at major steps and another one to small increments that depends on the functional clearance value (Figure 7). Dimensional chains for the case with two compensators are shown in Figure 8.

Realization of the process we will examine on example of precessional reducer (Figure 7). We admit, for example, that:

$$
A_{\Delta}=\sum_{i=1}^{n} T A_{i}=3.4 \cdot \mathrm{mm} .
$$

From dimensional chain (Figure 9, b) using the method of total interchangeability is determined the maximum value of clearance uncompensated by the previously compensator:

$$
J_{\max }^{N c o m p}=m_{2} \cdot \Delta k_{2}+J_{f \max }=m_{2} \cdot \Delta k_{2}+\left(J_{f}+\Delta k_{2}\right)=\left(m_{2}+1\right) \cdot \Delta k_{2}+J_{f}
$$

For various values of the steps number $m_{2}$ and value $\Delta k_{2}<J_{f}$. The value $\Delta k_{2}$ is expressed by $J_{f}$ :

$$
r=\Delta k_{2} / J_{f}
$$

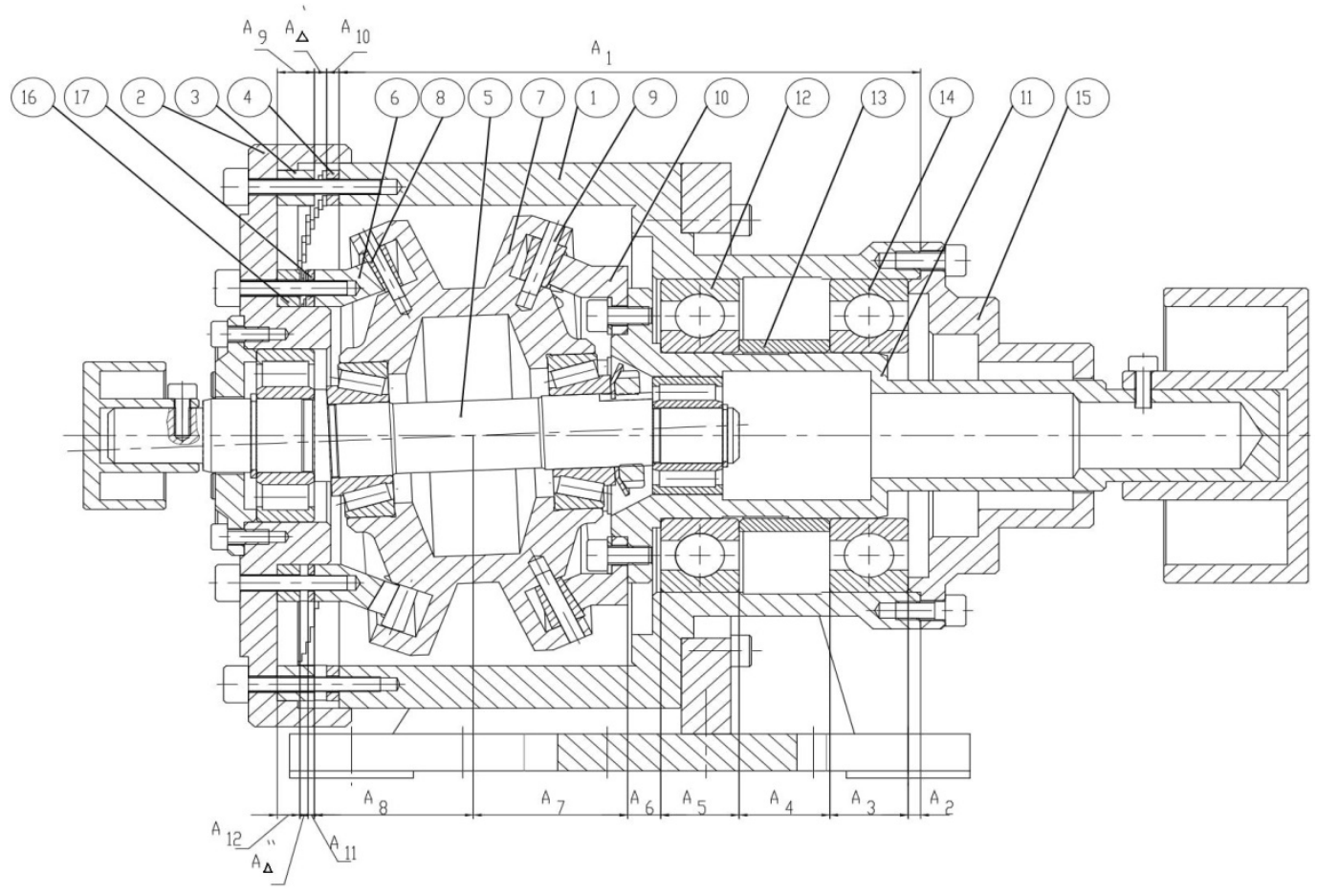

Fig. 7. Axial section for reducer with two compensators.

Then:

$$
J_{\max }^{N c o m p}=\left(m_{2}+1\right) \cdot \Delta k_{2}+J_{f}=J_{f} \cdot\left[\left(r \cdot\left(m_{2}+1\right)+1\right]\right.
$$


Is chosen one of the plausible obtained variants, for example: $m_{2}=7, J_{\max }^{N c o m p}=0.66$ for $r=0.7$. From dimensional chain (Figure 9, a) is determined the value of compensation step $\Delta k_{1}$ and the number of compensation steps, considering the fact that:

$$
J_{\max }^{N c o m p}=2 \cdot \Delta k_{1}
$$

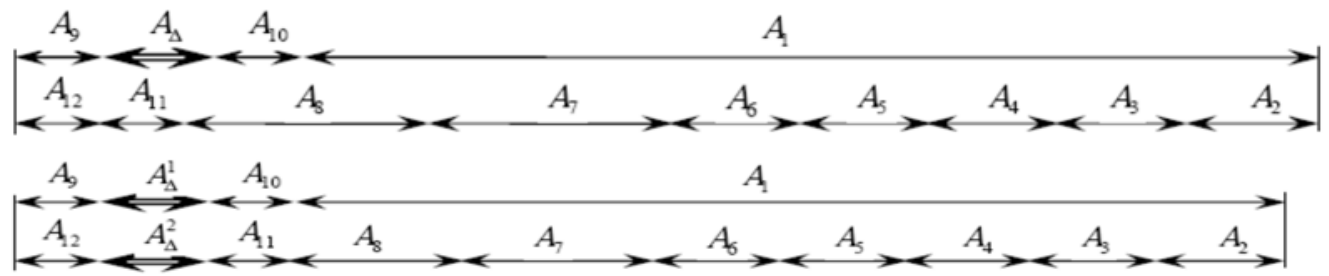

Fig. 8. Dimensional chains for the reducer with two compensators.

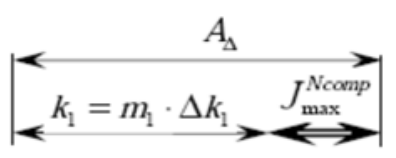

a)

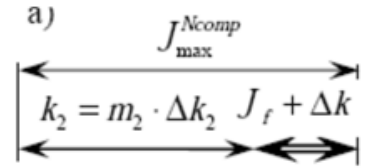

b)

Fig. 9. Dimensional chains of the compensators to ensure functional clearance.

Table 2. Obtained values.

\begin{tabular}{|c|c|c|c|}
\hline \multicolumn{2}{|c|}{$r=1$} & \multicolumn{2}{c|}{$r=0.7$} \\
\hline$m_{2}$ & $J_{\max }^{\text {Ncomp }}$ & $m_{2}$ & $J_{\max }^{\text {Ncomp }}$ \\
\hline 4 & 0.6 & 4 & 0.45 \\
\hline 5 & 0.7 & 5 & 0.52 \\
\hline 6 & 0.8 & 6 & 0.59 \\
\hline 7 & 0.9 & $\underline{7}$ & $\underline{0.66}$ \\
\hline 8 & 1 & 8 & 0.73 \\
\hline 9 & 1.1 & 9 & 0.8 \\
\hline 10 & 1.2 & 10 & 0.87 \\
\hline
\end{tabular}

$m_{1} \cdot \Delta k_{1}+J_{\max }^{N c o m p}=A_{\Delta} ;\left(m_{1}+2\right) \cdot \Delta k_{1}=A_{\Delta}$

$$
\Delta k_{1}=J_{\max }^{N c o m p} / 2=0.33
$$

$$
m_{1}=\frac{A_{\Delta}}{\Delta k_{1}}-2=\frac{3.4}{0.33}-2=8.3
$$

Is chosen $m_{1}=9$ and are recalculated the values Figure 10:

$$
\begin{gathered}
\Delta k_{1}=\frac{3.4}{9+2}=0.31, \\
J_{\max }^{N c o m p}=2 \cdot 0.31=0.62
\end{gathered}
$$


Is recalculated the value of functional clearance ensured by the second compensator:

$$
J_{f}=\frac{J_{\max }^{N c o m p}}{\left[\left(r \cdot\left(m_{2}+1\right)+1\right]\right.}=\frac{0.62}{0.7 \cdot(7+1)+1}=\frac{0.62}{6.6}=0.094
$$

The insured clearance is practically equal to the requested value $0.094 \approx 0.1$. For each compensator are determined according to compensated clearance the steps of compensation, uncompensated clearances and clearance deviations (see Table 1). If technical solution is not acceptable, recourse to another iteration operating with number of steps.
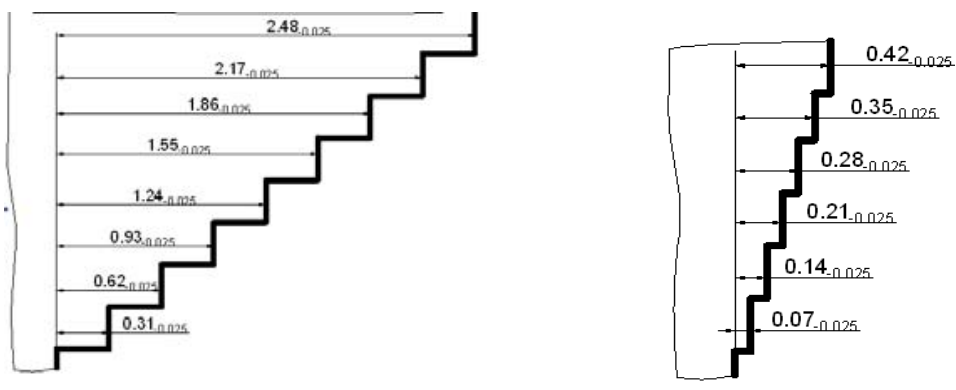

Fig. 10. Schemas with compensators stairs (example).
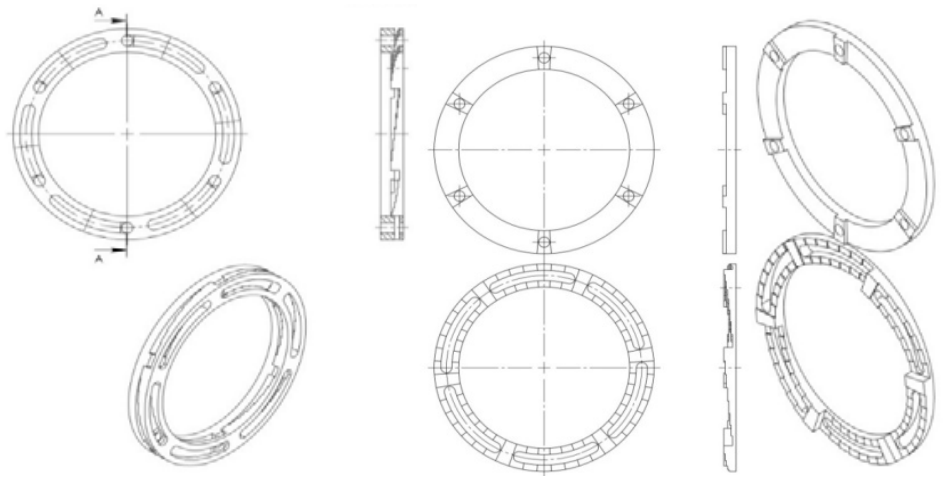

Fig. 11. Model of the compensator.

\section{Conclusions}

The minus of traditional method of clearance compensation is high workmanship of assembling, but the purpose of this method is to decrease the assembling costs and increasing of adjustment precision for axial clearance for different types of reducers, not only for precessional ones.

\section{References}

1. Iu. N. Berezovskii, N.A. Borodin, (1983)

2. V. Popa Ed. Tehnica-Info, Chişinău, (2006)

3. I.Bostan (in Russian), Chişinău, Science, (1991)

4. A. Toca, I. Stingaci and I. Rusica, App. Mech And Materials, ImanEE (2016) 